\title{
Antibiotic use for Vibrio infections: important insights from surveillance data
}

\author{
Kam Cheong Wong ${ }^{1,2}$, Anthony M. Brown², Georgina M. Luscombe², Shin Jie Wong ${ }^{1}$ and Kumara Mendis ${ }^{*}$
}

\begin{abstract}
Background: There is a paucity of data on the in vivo efficacy of antibiotics for lethal Vibrio species. Analyses of long-term surveillance datasets may provide insights into use of antibiotics to decrease mortality.

Methods: The United States Centers for Disease Control and Prevention (CDC) Cholera and Other Vibrio Illness Surveillance (COVIS) dataset from 1990 to 2010, with 8056 records, was analysed to ascertain trends in antibiotics use and mortality.

Results: Two-thirds of patients (5243) were prescribed antibiotics - quinolones (56.1\%), cephalosporins (24.1\%), tetracyclines (23.5\%), and penicillins (15.4\%). Considering all Vibrio species, the only class of antibiotic associated with reduced odds of mortality was quinolone (odds ratio $0.56,95 \% \mathrm{Cl}$ 0.46-0.67). Patients with V. vulnificus treated according to CDC recommendations had lower mortality (quinolone alone: 16.7 \%, $95 \%$ Cl 10.2-26.1; tetracycline plus cephalosporin: $21.7 \%$, 16.8-27.5; no antibiotic: $51.1 \%$, 45.6-56.7; each p <0.001). Cephalosporin alone was associated with higher mortality $(36.8 \%, 28.2-46.3)$. For $V$. cholerae non-O1, non-O139, mortality rates were lower for quinolone $(0 \%, 0-2.0)$ or tetracycline $(4.3 \%, 1.2-14.5)$ compared to no antibiotic $(9.3 \%, 6.4-13.3)$. For all Vibrio species, mortality rates increased with number of antibiotics in the treatment regimen $(p<0.001)$. Treatment regimens that included quinolone were associated with lower mortality rates regardless of the number of antibiotics used. The main clinical syndromes of patients with V. vulnificus infection were septicaemia (53.1\%) and wound infections (30.6\%). Mortality among $V$. vulnificus patients with septicaemia was significantly higher than for other clinical syndromes $(p<0.001)$. In a multivariate regression model, mortality in cases with $V$. vulnificus was associated with presence of pre-existing conditions (ORs ranged from 4.52 to 10.30), septicaemia (OR 2.64, $95 \% \mathrm{Cl} 1.92-3.63$ ) and no antibiotic treatment (OR 7.89, $95 \%$ Cl 3.94-15.80).
\end{abstract}

Conclusion: In view of the lack of randomized control trials, surveillance data may inform treatment decisions for potentially lethal Vibriosis. Considering all Vibrio species, use of quinolones is associated with lower mortality and penicillin alone is not particularly effective. For the most lethal species, $V$. vulnificus, treatment that includes either quinolone or tetracycline is associated with lower mortality than cephalosporin alone. We recommend treating patients who present with a clinical syndrome suggestive of $V$. vulnificus infection with a treatment regimen that includes a quinolone.

Keywords: Vibrio infection, Vibriosis, Antibiotics, Antimicrobials, Surveillance

\footnotetext{
*Correspondence: k.mendis@uws.edu.au

${ }^{1}$ Bathurst Rural Clinical School, School of Medicine, University of Western

Sydney, PO Box 9008, Bathurst, NSW 2795, Australia

Full list of author information is available at the end of the article
} 


\section{Background}

The incidence of Vibrio infections, which can cause acute diarrhoea and potentially serious complications such as hypovolemic shock and septicaemia, continues to rise in the United States [1]. Vibrio vulnificus is the most lethal species, and there are limited data on the effectiveness of antibiotic use in $V$. vulnificus infections [2]. In 1988, the Centers for Disease Control and Prevention $(\mathrm{CDC})$ in the United States established a surveillance system for human infections caused by all species of Vibrio known as "Cholera and Other Vibrio Illness Surveillance" (COVIS) [3]. This surveillance information has been used to inform healthcare providers and educate the public [4].

Clinical trials indicate that using an effective antibiotic as an adjuvant therapy to treat $V$. cholerae reduces the duration of diarrhoea $[5,6]$ and illness by almost $50 \%$ in patients with moderate and severe dehydration [6]. Studies of treatment efficacy for lethal Vibrio species such as $V$. vulnificus, have inherent ethical difficulties and consequently there are no randomised control trials (RCT) of $V$. vulnificus in humans [2]. Animal model in vivo studies of antibiotic sensitivities to Vibrio infection may not all be applicable to humans due to differences in pharmacokinetic parameters [7]. Recommendations for $V$. vulnificus infections from a recent review are largely based on case reports or animal models [8]. The CDC provides recommendations on treatment regimens for $V$. vulnificus, $V$. parahaemolyticus and $V$. cholerae non-O1, non-O139 [4]. However only recommendations on treating $V$. cholerae are based on a human RCT [4].

Observational studies on lethal Vibrio infections, using established surveillance data over a long defined period of time, may provide insights about the associations between the use of antibiotics and patient outcomes. For example, Purcell et al. conducted a systematic review of the use of prophylactic antibiotics in the prevention of meningococcal disease and had to rely almost entirely on surveillance data to support their use [9]. Dechet and colleagues reviewed non-foodborne Vibrio infections using COVIS data (1997 to 2006) and concluded that the optimal antibiotic treatment for Vibrio infections remains unknown [10].

We have analysed COVIS data from 1990 to 2010: (a) to ascertain whether the COVIS data on antibiotic use were consistent with the $\mathrm{CDC}$ treatment recommendations, and (b) to determine the relationship between antibiotic treatment and mortality.

\section{Methods}

\section{Study design and participants}

Data were obtained from the CDC's Enteric Diseases Epidemiology Branch from case report forms submitted to the COVIS during 1990 to 2010 [1]. COVIS data are collected using a standardised form [3]. The COVIS dataset included information on demographics, the Vibrio species isolated and the source of the specimen, clinical features (symptoms and signs), mortality, and pre-existing conditions and treatments during the 30 days prior to the Vibrio illness. The dataset also included information on whether or not the patient had an antibiotic as treatment for the Vibrio illness (i.e. yes, no, unknown), and if so, the name of the antibiotic/s.

Free text records of antibiotic names and some preexisting conditions and treatments (specifically malignancy, immune disorders, proton pump inhibitors and antacids) were reviewed by authors KCW and SJW independently and classified using the Systematized Nomenclature of Medicine - Clinical Terms (SNOMED $\mathrm{CT}^{\circ}$ ) system (SNOMED Premium Version 1.0) and then reviewed jointly to reach consensus [11]. Pre-existing conditions included heart disease, diabetes, liver disease, alcoholism, malignancy, renal disease, haematological disease and immune disorders. SNOMED $\mathrm{CT}^{\circ}$ codes were used to classify antibiotics into antimicrobial classes (e.g. quinolone, cephalosporin), antimicrobial subclasses (e.g. first, second and third generation cephalosporins) and to standardise and review pre-existing conditions and treatments (e.g. free text records of gastric surgery were reviewed and those considered irrelevant, such as hernia repair, were removed).

There were 8950 patients in the COVIS dataset, 6137 (68.6\%) of whom were recorded as having had antibiotics as treatment for their Vibrio illness. However, the specific name of the antibiotic was not recorded for 894 (14.6\%). A sensitivity analysis, whereby results were compared for analyses which either included or excluded the 894 patients who had an unnamed antibiotic for the Vibrio illness, was performed. There were no substantial differences in terms of their epidemiological information and mortality rates, and consequently the patients without a named antibiotic were excluded from subsequent analyses, resulting in a final sample size of $\mathrm{N}=8056$.

\section{Statistical analysis}

For the purposes of analysis, where antibiotic use was recorded in COVIS as 'unknown' this was recoded as 'no' antibiotic used. Similarly, where data were missing or recorded as 'unknown' for clinical signs or symptoms, pre-existing conditions or treatments, or mortality, they were recoded as 'absent' or 'no' for analysis. Based on reported symptoms, patients with $V$. vulnificus were classified into the following clinical syndrome groups: (i) septicaemia, characterised by the isolation of the organism from blood AND the presence of either fever or shock; (ii) gastroenteritis, defined as the presence of blood in stool, OR both diarrhoea and vomiting, OR any gastrointestinal symptom AND the isolation of organism 
from stool; (iii) wound infection, characterised by cellulitis OR bullae OR fever in the absence of septicaemia, where the organism was isolated from a wound only; or (iv) other (not meeting any of the other criteria). Where a patient met the criteria for more than one clinical syndrome, septicaemia took precedence over gastroenteritis, and gastroenteritis over a wound infection.

Skewed continuous data were reported as medians with an interquartile range (IQR). Associations between demographics, antibiotic use and mortality were explored using unadjusted odds ratios with $95 \%$ confidence intervals. Chi-square analyses explored univariate associations between factors such as antibiotic use and mortality. The association between the total number of antibiotics used and mortality was explored using the linear-by-linear association chi-square test. A series of univariate logistic regression models were conducted on the sub-sample of patients with $V$. vulnificus to determine predictors of mortality. Variables in these regression analyses included age, gender, year of notification, number of pre-existing conditions, clinical syndrome presentation and type of antibiotic regimen (quinolone only; quinolone and another antibiotic; at least one antibiotic, but not quinolone; or no antibiotic at all). Unadjusted odds ratios (OR) and $95 \%$ confidence intervals (CIs) were produced. A multivariate logistic regression analysis was also conducted, including all of these variables, to produce adjusted ORs and $95 \%$ CIs.

All analyses were conducted using SPSS (version 21; IBM, 2012) and $\alpha$ was set at $\mathrm{p}<0.05$.

\section{Ethics committee approval}

All data were de-identified and the CDC confirmed that ethics approval and informed consent were not applicable for this research.

\section{Results}

Of the 8056 patients, the median age was 47 years (IQR 33 to 62 years; $\mathrm{N}=7773$ ). Over two-thirds of the patients were male $(68.6 \%$; 5424/7905) and $98.3 \%$ (7921/8056) of the patients had only one Vibrio species identified. 127 $(1.6 \%)$ patients had two species and eight $(0.1 \%)$ patients had three Vibrio species identified. The most commonly identified species were $V$. parahaemolyticus, $V$. vulnificus, $V$. alginolyticus and the non-O group strains of $V$. cholerae (Table 1). The proportion of fatal cases differed by Vibrio species, the greatest being in those with $V$. vulnificus (Table 1). Cases of V. parahaemolyticus increased dramatically over time, with peaks in 1998, 2004, 2006 and 2009-10, and there was a steady increase in cases of $V$. alginolyticus (Fig. 1). Peak incidence typically occurred during the summer months (June to August, $52.6 \%$ of cases).
Table 1 Vibrio species, mortality and gender distribution; United States cases during 1990 to $2010(N=8056)$

\begin{tabular}{|c|c|c|c|}
\hline Vibrio species & Total n (\%) & Mortality n (\%) & Male $\mathrm{n}(\%)$ \\
\hline V. parahaemolyticus & $3474(43.1)$ & $29(0.8)$ & $2243(66.1)$ \\
\hline V. vulnificus & 1599 (19.8) & $491(30.7)$ & $1367(86.4)$ \\
\hline V. alginolyticus & $874(10.8)$ & $11(1.3)$ & $589(69.1)$ \\
\hline V. cholerae non-O1, non-O139 & $763(9.5)$ & $41(5.4)$ & $474(62.9)$ \\
\hline V. fluvialis & $445(5.5)$ & $13(2.9)$ & $243(56.0)$ \\
\hline V. cholerae 01 & $269(3.3)$ & $4(1.5)$ & $137(51.1)$ \\
\hline V. mimicus & $211(2.6)$ & $5(2.4)$ & $116(55.0)$ \\
\hline V. hollisae ${ }^{a}$ & $131(1.6)$ & $1(0.8)$ & $79(61.2)$ \\
\hline P. damsela ${ }^{b}$ & $63(0.8)$ & $3(4.8)$ & $45(71.4)$ \\
\hline V. cholerae 0139 & $28(0.3)$ & $0(0.0)$ & $15(57.7)$ \\
\hline V. furnissii & $19(0.2)$ & $1(5.3)$ & $13(76.5)$ \\
\hline V. metschnikovii & $14(0.2)$ & $1(7.1)$ & $7(53.8)$ \\
\hline V. cincinnatiensis & $2(<0.1)$ & $0(0.0)$ & $1(50.0)$ \\
\hline Species not identified & $279(3.5)$ & $9(3.2)$ & $169(61.7)$ \\
\hline Other, no further information & $28(0.3)$ & $0(0.0)$ & $16(64.0)$ \\
\hline Total & $\mathrm{n} / \mathrm{a}$ & $602(7.5)$ & 5424 (67.3) \\
\hline
\end{tabular}

N.B. some patients had more than one species isolated, so numbers total to $\mathrm{N}=8199$

Proportions here are of the total patients, not total species identified

Information on gender was missing for $\mathrm{n}=151$

${ }^{a}$ Formerly Vibrio hollisae, now Grimontia hollisae [2]

${ }^{b}$ Formerly Vibrio damsela, now Photobacterium damsela

There were $5243(65.1 \%)$ patients who had an antibiotic as treatment for their Vibrio illness. The most commonly used antibiotics were quinolones (56.1\%), followed by cephalosporins (24.1\%), tetracyclines (23.5 \%) and penicillins $(15.4 \%)$. The use of quinolones increased considerably after 1996 with peaks around 1997, 2005, and 2010, while the use of cephalosporin, tetracycline and penicillin rose only slowly from 1990 to 2010 (Fig. 2).

A total of $602(7.5 \%)$ patients with a Vibrio illness were recorded as deceased. Mortality was significantly associated with being male (OR 2.10, 95 \% CI 1.70-2.59). Bullae (present in $7.3 \%$ of cases), and shock ( $6.5 \%)$ were associated with significantly increased odds of mortality (OR 8.67, 95 \% CI 7.11-10.58; OR 28.49, 95 \% CI 23.1835.01 respectively). Presence of at least one of the eight pre-existing conditions $(33.2 \%)$ significantly increased risk of death (OR 16.83, $95 \%$ CI 13.18-21.49); in particular, a history of liver disease (10.6\%; OR 18.81, $95 \%$ CI 15.63-22.63) and alcoholism (8.9\%; OR 13.50, $95 \%$ CI 11.19-16.27).

The association between antibiotic classes (use of at least one antibiotic of a particular class in a treatment regimen) and mortality is presented by Vibrio species in Table 2. Considering all patients, regardless of Vibrio species, the only class of antibiotic associated with reduced odds of mortality was quinolone (OR 0.56, $95 \%$ 


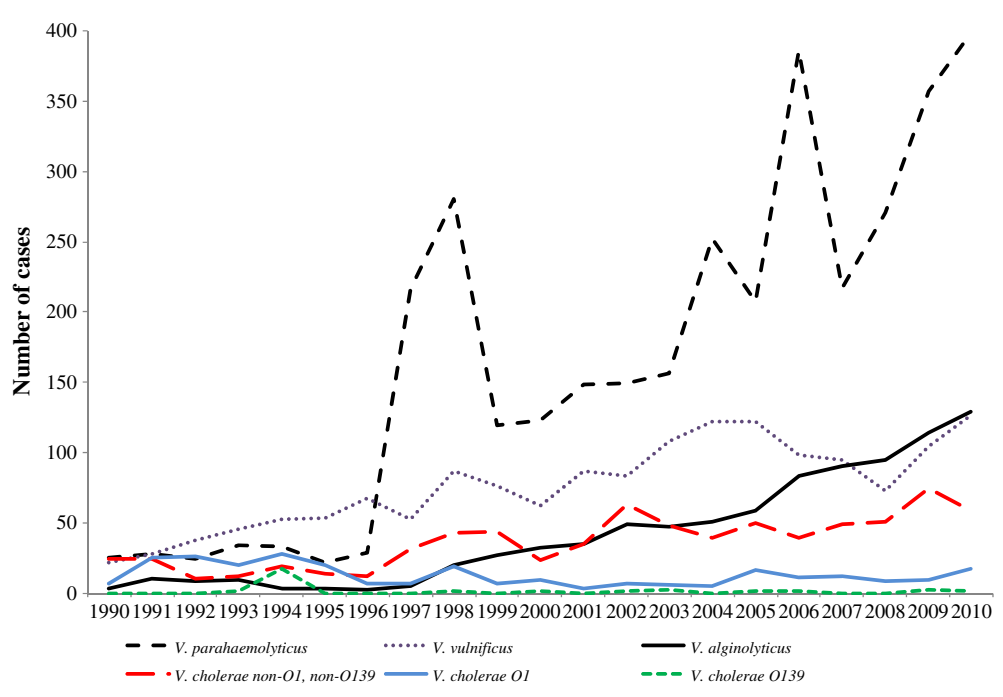

Fig. 1 Patients with Vibrio infections over time, 1990 - 2010, in United States

CI 0.46-0.67). This association held for those patients with $V$. vulnificus (OR 0.58, $95 \%$ CI 0.46-0.73), and $V$. cholerae (non-O1, non-O139) (OR 0.12, 95 \% CI 0.040.40). Cephalosporin was associated with an increased odds of mortality (OR 2.60, $95 \%$ CI 2.16-3.13) overall, and notably for $V$. parahaemolyticus (OR 6.41, $95 \%$ CI 2.89-14.24). For those patients with $V$. vulnificus infection, use of the antibiotics quinolone, cephalosporin and tetracycline was associated with significantly lower mortality rate while use of penicillin was equivocal.

There was a statistically significant positive association between the mortality rates and the number of antibiotics used in a treatment regimen (linear by linear association $=172.90, \mathrm{p}<0.001$ ). Irrespective of Vibrio species, a treatment regimen that included a quinolone was always associated with lower mortality rate regardless of the total number of antibiotics in the treatment regimen (Fig. 3a). Whether or not the treatment regimen included a cephalosporin, tetracycline, or penicillin was not associated consistently with the mortality rate (Fig. 3b, c, d).

Tables 3 and 4 show the mortality associated with various antibiotic regimens (including CDC recommendations) for treating Vibrio vulnificus and Vibrio cholerae non-O1, non-O139 infections. Mortality rates for $V$. vulnificus were significantly lower in those patients taking quinolone only or tetracycline combined with a third generation cephalosporin as per CDC recommendations (Table 3). Using quinolone as a reference, comparison between the CDC recommended combination (i.e. tetracycline and third generation cephalosporin) and other combinations that included quinolone, showed no statistically significant differences, except the comparison with taking a cephalosporin alone or a penicillin alone or no antibiotic $(\mathrm{p}=0.002, \mathrm{p}=0.024, \mathrm{p}<0.001$ respectively; Table 3). For $V$. cholerae (non O1 and non-O139), only use of quinolone alone was associated with a significantly lower mortality rate (Table 4).

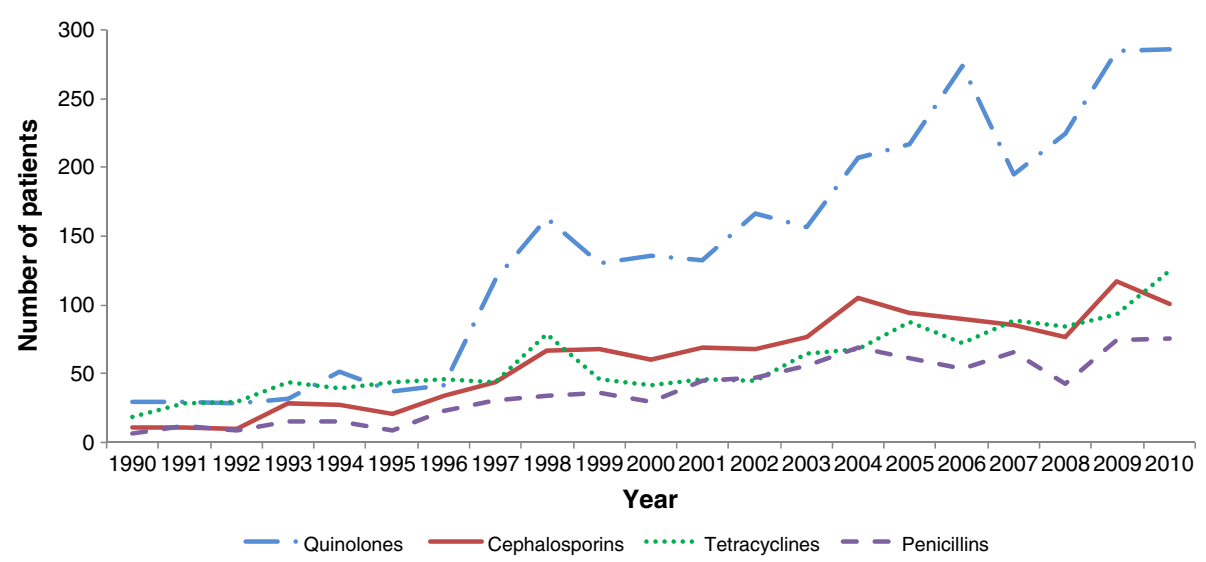

Fig. 2 Patterns of antibiotic use in the United States for patients with Vibrio illness: 1990 to 2010 ( $N=5243$ ) 
Table 2 Mortality and antibiotic use, by type of Vibrio illness $(\mathrm{N}=8056)$

\begin{tabular}{|c|c|c|c|}
\hline All Vibrio species & $\begin{array}{l}\text { Alive } \\
\mathrm{n}=7454\end{array}$ & $\begin{array}{l}\text { Deceased } \\
\mathrm{n}=602\end{array}$ & $\begin{array}{l}\text { Crude Odds Ratio } \\
(95 \% \mathrm{Cl})\end{array}$ \\
\hline $\begin{array}{l}\text { Received at least one } \\
\text { antibiotic }\end{array}$ & $65.1 \%$ & $64.6 \%$ & $0.98(0.82-1.16)$ \\
\hline At least one quinolone & $37.4 \%$ & $24.9 \%$ & $0.56(0.46-0.67)$ \\
\hline $\begin{array}{l}\text { At least one } \\
\text { cephalosporin }\end{array}$ & $14.5 \%$ & $30.6 \%$ & $2.60(2.16-3.13)$ \\
\hline At least one tetracycline & $14.5 \%$ & $25.1 \%$ & $1.98(1.63-2.40)$ \\
\hline At least one penicillin & $9.5 \%$ & $15.8 \%$ & $1.78(1.41-2.25)$ \\
\hline V. parahaemolyticus & $n=3445$ & $n=29$ & \\
\hline $\begin{array}{l}\text { Received at least one } \\
\text { antibiotic }\end{array}$ & $56.5 \%$ & $65.5 \%$ & $1.46(0.68-3.15)$ \\
\hline At least one quinolone & $39.8 \%$ & $37.9 \%$ & $0.93(0.44-1.97)$ \\
\hline $\begin{array}{l}\text { At least one } \\
\text { cephalosporin }\end{array}$ & $6.6 \%$ & $31.0 \%$ & $6.41(2.89-14.24)$ \\
\hline At least one tetracycline & $7.3 \%$ & $20.7 \%$ & $3.29(1.33-8.16)$ \\
\hline At least one penicillin & $4.6 \%$ & $3.4 \%$ & $0.75(0.10-5.53)$ \\
\hline V. vulnificus & $\mathrm{n}=1108$ & $n=491$ & \\
\hline $\begin{array}{l}\text { Received at least one } \\
\text { antibiotic }\end{array}$ & $86.6 \%$ & $68.2 \%$ & $0.33(0.26-0.43)$ \\
\hline At least one quinolone & $37.1 \%$ & $25.5 \%$ & $0.58(0.46-0.73)$ \\
\hline $\begin{array}{l}\text { At least one } \\
\text { cephalosporin }\end{array}$ & $42.3 \%$ & $33.2 \%$ & $0.68(0.54-0.85)$ \\
\hline At least one tetracycline & $47.5 \%$ & $27.3 \%$ & $0.42(0.33-0.52)$ \\
\hline At least one penicillin & $18.5 \%$ & $17.7 \%$ & $0.95(0.72-1.25)$ \\
\hline V. alginolyticus & $n=863$ & $\mathrm{n}=11$ & \\
\hline $\begin{array}{l}\text { Received at least one } \\
\text { antibiotic }\end{array}$ & $76.0 \%$ & $36.4 \%$ & $0.18(0.05-0.62)$ \\
\hline At least one quinolone & $29.7 \%$ & $18.2 \%$ & $0.53(0.11-2.46)$ \\
\hline $\begin{array}{l}\text { At least one } \\
\text { cephalosporin }\end{array}$ & $23.6 \%$ & $18.2 \%$ & $0.72(0.15-3.35)$ \\
\hline At least one tetracycline & $10.0 \%$ & $0.0 \%$ & $\mathrm{n} / \mathrm{a}$ \\
\hline At least one penicillin & $19.1 \%$ & $9.1 \%$ & $0.42(0.05-3.33)$ \\
\hline $\begin{array}{l}\text { V. cholerae non-O1, non- } \\
\text { O139 }\end{array}$ & $n=722$ & $n=41$ & \\
\hline $\begin{array}{l}\text { Received at least one } \\
\text { antibiotic }\end{array}$ & $66.1 \%$ & $39.0 \%$ & $0.33(0.17-0.63)$ \\
\hline At least one quinolone & $39.3 \%$ & $7.3 \%$ & $0.12(0.04-0.40)$ \\
\hline $\begin{array}{l}\text { At least one } \\
\text { cephalosporin }\end{array}$ & $9.4 \%$ & $19.5 \%$ & $2.33(1.04-5.25)$ \\
\hline At least one tetracycline & $12.3 \%$ & $19.5 \%$ & $1.72(0.77-3.85)$ \\
\hline At least one penicillin & $9.6 \%$ & $7.3 \%$ & $0.75(0.23-2.48)$ \\
\hline
\end{tabular}

n/a not applicable

Considering only $V$. vulnificus, $53.1 \%(839 / 1581)$ had septicaemia, $4.5 \%$ (71) had gastroenteritis, $30.6 \%$ (484) had a wound infection, and $11.8 \%$ (187) had other clinical presentation. Eighteen cases were excluded because they could not be classified as information on the sample source was missing. Cases with septicaemia were more likely to fatal $(40.6 \%, 341 / 839)$, followed by $28.9 \%$ (54) of those with other presentation, $28.2 \%$ (20) of those with gastroenteritis and $14.3 \%$ (69) of those with wound infections $(p<0.001)$. A series of logistic regression analyses with single predictor variables was conducted, with year of notification dichotomised into 1990 to 1996 versus 1997 to 2010 to reflect the significant increase in quinolone use from 1996 observed in Fig. 2. The crude odds of mortality amongst those with $V$. vulnificus increased significantly with the number of preexisting conditions (see Table 5). In comparison to wound infections, all other clinical syndromes conferred increased odds of dying. Compared to quinolone alone, an antibiotic regimen without quinolone, and no antibiotic use at all were associated with increased odds of mortality. The multivariate analysis on mortality for patients with $V$. vulnificus infection showed the same patterns for number of pre-existing conditions. Gastroenteritis no longer conferred significantly greater odds of dying over wound infections, but septicaemia and other clinical syndrome did. In the adjusted model, quinolone alone remained superior in comparison to no antibiotic treatment.

\section{Discussion}

Using this large surveillance dataset covering more than two decades, we determined that the two most important factors associated with mortality are the particular Vibrio species and the class of antibiotic used. Quinolone is the only class of antibiotic associated with lower mortality in all Vibrio species, regardless of the number of antibiotics used in a treatment regimen. In potentially lethal $V$. vulnificus infections, the use of quinolone alone or the combination of tetracycline and third generation cephalosporin had the lowest mortality which is in-line with the current $\mathrm{CDC}$ recommendations.

Penicillin was the least effective antibiotic for vibriosis according to our analysis. In 1984, the USA National Institutes of Health recommended use of penicillin or tetracycline to treat $V$. vulnificus based on in vitro sensitivity studies [12]. Morris cautioned that in vitro data can be misleading, and recommended against using penicillin as a single antibiotic to treat $V$. vulnificus infections in humans [13]. In 2002, Tang conducted a study on mice and reported that quinolones as single antibiotics were as effective as cefotaxime-minocycline (third generation cephalosporin and tetracycline) in combination [7]. However Tang cautioned that results of animal models may not be applicable to humans due to differences in pharmacokinetic parameters [7]. Our findings support Morris's caution against penicillin as a single antibiotic (i.e. penicillin was not associated with reduced mortality) and substantiate Tang's previous findings in animals (i.e. third generation cephalosporin and 

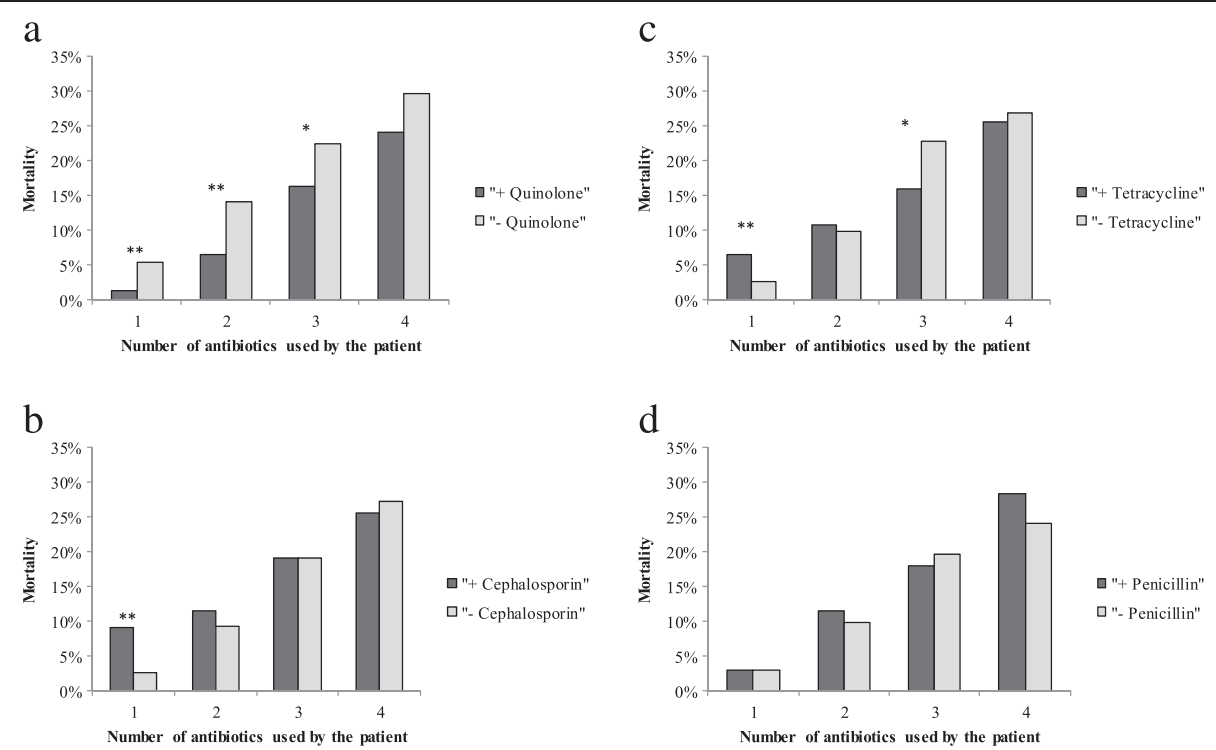

Fig. 3 a Mortality by use of quinolone in treatment regimen. b Mortality by use of cephalosporin in treatment regimen. c Mortality by use of tetracycline in treatment regimen. $\mathbf{d}$ Mortality by use of penicillin in treatment regimen. ${ }^{*}$ indicates a statistically significant difference at $p<$ $0.001,{ }^{*}$ indicates a statistically significant difference at $p<0.05 . n=3132$ used one antibiotic; 1305 used two antibiotics; 652 used three antibiotics; 154 used four antibiotics

tetracycline in combination are more effective). More recently Shaw and colleagues evaluated the antimicrobial susceptibility of $V$. vulnificus recovered from two commercial environmental areas and found that $V$. vulnificus demonstrated resistance to penicillin [14], which may further confirm the inefficacy of penicillin.

For $V$. cholerae (non-O1, non-O139), quinolone was the only class of antibiotic associated with lower mortality rate. For $V$. parahaemolyticus, quinolone and penicillin appeared to have equivocal odds ratios for mortality; while cephalosporin and tetracycline were associated with higher mortality. However, the total number of deaths from $V$. cholerae was 41 and for $V$. parahaemolyticus was 29 , so conclusions are limited.

Generally, the mortality rate increased along with the number of antibiotics used in the treatment regimen. We postulated that seriously ill patients were given more than one antibiotic and were associated with increased

Table 3 Mortality associated with various antibiotic regimens in the treatment of $\mathrm{V}$. vulnificus ( $\mathrm{n}=1599)$

\begin{tabular}{|c|c|c|c|}
\hline & $\mathrm{N}^{\mathrm{a}}$ & Crude mortality \% (95\% Cl) & Comparison with Quinolone only p value \\
\hline \multicolumn{4}{|l|}{ CDC treatment recommendation ${ }^{\mathrm{b}}$} \\
\hline Quinolone only & $14 / 84$ & $16.7(10.2-26.1)$ & - \\
\hline Tetracycline + cephalosporin (all generations) & $49 / 226$ & $21.7(16.8-27.5)$ & 0.329 \\
\hline Tetracycline $+3^{\text {rd }}$ generation cephalosporin & $39 / 182^{c}$ & $21.4(16.1-28.0)$ & 0.366 \\
\hline \multicolumn{4}{|l|}{ Other combinations } \\
\hline Quinolone + cephalosporin (all generations) & $24 / 98$ & $24.5(17.1-33.9)$ & 0.195 \\
\hline Quinolone + tetracycline & $15 / 95$ & $15.8(9.8-24.4)$ & 0.874 \\
\hline Quinolone + cephalosporin + tetracycline & $15 / 87$ & $17.2(10.7-26.5)$ & 0.920 \\
\hline \multicolumn{4}{|l|}{ Other single antibiotics } \\
\hline Tetracycline alone & $30 / 145$ & $20.7(14.9-28.0)$ & 0.456 \\
\hline Cephalosporin alone (all generations) & $39 / 106$ & $36.8(28.2-46.3)$ & 0.002 \\
\hline Penicillin alone & $18 / 54$ & $33.3(22.2-46.6)$ & 0.024 \\
\hline No antibiotic & $156 / 305$ & $51.1(45.6-56.7)$ & $<0.001$ \\
\hline
\end{tabular}

${ }^{a}=399$ patients with less common antibiotic regimens not included here, thus numbers do not total to $n=1599$

b http://www.cdc.gov/vibrio/vibriov.html (accessed 27 June 2014): “Doxycycline (100 mg PO/IV twice a day for 7-14 days) and a third-generation cephalosporin (e.g. ceftazidime 1-2 g IV/IM every eight hours) are generally recommended. A single agent regimen with a fluoroquinolone such as levofloxacin, ciprofloxacin or gatifloxacin, has been reported to be at least as effective in an animal model as combination drug regimens with doxycycline and a cephalosporin"

$c_{n}=182$ patients are a subset of the $n=226$ patients with a tetracycline combined with a cephalosporin 
Table 4 Mortality associated with various antibiotic regimens in the treatment of $V$. cholerae non-O1, non-0139 $\left(n=763^{\mathrm{a}}\right)$

\begin{tabular}{lll}
\hline $\mathrm{N}$ & $\begin{array}{l}\text { Crude mortality \% } \\
(95 \% \mathrm{Cl})\end{array}$ & $\begin{array}{l}\text { Comparison with } \\
\text { Tetracycline alone } \\
\end{array}$ \\
& FET p value
\end{tabular}

CDC treatment

recommendation ${ }^{b}$

Tetracycline alone $\quad 2 / 46 \quad 4.3(1.2-14.5)$

Other single antibiotics

$\begin{array}{lccc}\text { Quinolone alone } & 0 / & 0.0(0.0-2.0) & 0.037 \\ & 192 & & \\ \text { Penicillin alone } & 1 / 38 & 2.6(0.5-13.5) & 1.000 \\ \text { Cephalosporin alone } & 2 / 29 & 6.9(1.9-22.0) & 0.638 \\ \text { antibiotic } & 25 / & 9.3(6.4-13.3) & 0.395 \\ & 270 & & \end{array}$

FET fisher's exact test

${ }^{a} n=188$ patients with less common antibiotic regimens not included here, thus numbers do not total to $n=763$

${ }^{b}$ http://www.cdc.gov/cholera/treatment/antibiotic-treatment.html (accessed 27 June 2014): "Antibiotic choices should be informed by local antibiotic susceptibility patterns. In most countries, doxycycline is recommended as first-line treatment for adults, while azithromycin is recommended as first-line treatment for children and pregnant women" number of antibiotics in their treatment; hence, their mortality rate was higher possibly because they were sicker instead of due to the larger number of antibiotics used. We found that quinolone was the only antibiotic associated with reduced mortality rate regardless of the number of antibiotics in the patient's regimen.

We have shown an increase in use of quinolone after 1996 but this may be a reflection of increased use in the wider community. Linder and colleagues have reported a three-fold increase in prescribing quinolone between 1995 and 2005 in the United States adult population [15]. This increase in quinolone prescription may not necessarily reflect increased recognition of the efficacy of this antibiotic amongst prescribers or the promulgation of treatment guidelines. It is likely that marketing, advertising and provision of sample antibiotics might have partly contributed to the increase in prescribing of newer antibiotics [15-17].

We found associations between liver disease, alcoholism and previous ill health and mortality with all species. For $V$. vulnificus the number of pre-existing conditions was associated with increased odds of mortality in both unadjusted and adjusted models. Others have found that patients with liver disease or alcoholism are at higher risk of $V$. vulnificus infection $[10,18,19]$. This may be

Table 5 Predictors of death in cases of V. vulnificus in the United States during 1990 to 2010

\begin{tabular}{|c|c|c|c|c|}
\hline & Fatal N (\%) & Non-fatal N (\%) & Crude OR (95\% Cl) & Adjusted OR (95\% Cl) \\
\hline Age, mean (SD) & $56.6(13.5)$ & $58.0(17.8)$ & $0.995(0.989-1.001)$ & $0.99(0.98-0.998)$ \\
\hline \multicolumn{5}{|l|}{ Gender } \\
\hline Female & $72(15.2)$ & 135 (12.6) & reference & reference \\
\hline Male & $402(84.8)$ & $939(87.4)$ & $0.80(0.59-1.09)$ & $0.70(0.49-1.000)$ \\
\hline \multicolumn{5}{|l|}{ Year of notification } \\
\hline $1990-1996$ & $103(21.7)$ & $194(18.1)$ & reference & reference \\
\hline $1997-2010$ & $371(78.3)$ & $880(81.9)$ & $0.79(0.61-1.04)$ & $0.99(0.73-1.34)$ \\
\hline \multicolumn{5}{|l|}{ Pre-existing conditions } \\
\hline 0 & $41(8.6)$ & $397(37.0)$ & reference & reference \\
\hline 1 & 107 (22.6) & $277(25.8)$ & $3.74(2.53-5.53)$ & $4.52(2.92-6.99)$ \\
\hline 2 & $194(40.9)$ & $227(21.1)$ & $8.28(5.69-12.03)$ & $10.30(6.72-15.78)$ \\
\hline 3 or more & $132(27.8)$ & $173(16.1)$ & 7.39 (4.99-10.95) & $9.31(5.93-14.62)$ \\
\hline \multicolumn{5}{|l|}{ Clinical presentation } \\
\hline Wound & $68(14.3)$ & 406 (37.8) & reference & reference \\
\hline Gastroenteritis & $20(4.2)$ & $49(4.6)$ & $2.44(1.36-4.35)$ & $1.52(0.77-3.01)$ \\
\hline Septicaemia & $333(70.3)$ & $493(45.9)$ & $4.03(3.01-5.40)$ & $2.64(1.92-3.63)$ \\
\hline Other & $53(11.2)$ & $126(11.7)$ & $2.51(1.67-3.79)$ & $1.69(1.04-2.74)$ \\
\hline \multicolumn{5}{|l|}{ Antibiotic treatment } \\
\hline Quinolone only & $13(2.7)$ & $68(6.3)$ & reference & reference \\
\hline Quinolone and other/s & $106(22.4)$ & $335(31.2)$ & $1.66(0.88-3.12)$ & $1.44(0.73-2.84)$ \\
\hline Antibiotic/s, not quinolone & $208(43.9)$ & $534(49.7)$ & $2.04(1.10-3.77)$ & $1.84(0.95-3.56)$ \\
\hline None & $147(31.0)$ & $137(12.8)$ & $5.61(2.97-10.62)$ & $7.89(3.94-15.80)$ \\
\hline
\end{tabular}


because $V$. vulnificus uses transferrin-bound iron, which is usually abundant in these patients, for growth [18, 20]. Another hypothesis about the increase mortality in the presence of liver disease is that the shunting of portal blood containing V. vulnificus infection around a diseased liver may lead to septicaemia [21]. The main clinical syndromes in patients with $V$. vulnificus infection were septicaemia and wound infections. Mortality among $V$. vulnificus patients with septicaemia was significantly higher than for other clinical syndromes. A treatment regimen that included quinolone was associated with lower mortality compared with cephalosporin alone or penicillin alone or no antibiotic at all. We recommend that patients who present with a clinical syndrome suggestive of $V$. vulnificus infection be treated with a regimen that includes a quinolone.

There are a number of limitations to this study that relate to the underlying surveillance data collection. For example, non-cholera vibriosis only became nationally notifiable in USA from 2007 onwards [22]. Several pertinent details regarding use of antibiotics were not recorded systematically such as timing or order of antibiotic use. There were 894 patients who had an unnamed antibiotic for the Vibrio infection. Cephalosporins could not be categorised into the four generations due to the small subgroup size. This may have contributed to the paradoxical observation in Table 2 where a treatment regimen that had included a cephalosporin was associated with increased mortality rate in $V$. cholera (non-O1 and non-O139) and V. parahaemolyticus infections, but reduced mortality elsewhere. The dataset did not detail specific cause of death, but an infection such as Vibrio is likely to have been significant. Further characterisation of liver disease (type and severity) was not possible due to the inconsistency of these data in the COVIS dataset. Finally, it is possible that the use and effectiveness of different antibiotics reflect changes in the antibiotic sensitivities of the organism, however we are unable to explore this with the data available in the COVIS dataset.

Determining the optimal antibiotic regimen for a potentially lethal infection is difficult because randomised controlled trials may not be possible and in vitro or animal models may not be easily applied in patients. In this context, systematic experience from detailed surveillance data may inform treatment decisions. However information from surveillance data is only as good as the data collected. The COVIS dataset may not have included every Vibrio cases and reporting may have been biased towards more severe cases [22]. It is important that clinicians provide detailed and timely data to surveillance programs such as COVIS. Publication of findings from surveillance data may help encourage clinicians to provide specific data regarding antibiotic use. Surveillance authorities must be encouraged to simplify and refine the data collection tools and seek more specific information on classes of antibiotics and the time frame of their use so that the influence of these important factors can be reported and analysed.

\section{Conclusions}

Surveillance of large numbers of affected individuals over longer periods of time appears to be a reasonable method of determining antibiotic use and outcome patterns. Vibrio infection remains a serious condition with significant mortality. Adjuvant antibiotic therapy in addition to basic care with fluids has an important role. The use of quinolones may reduce risk of death in patients with $V$. vulnificus and $V$. cholerae (non-O1 and non-O139). For $V$. vulnificus, which has the highest mortality rate, a treatment regimen which is in line with the CDC recommendations i.e. that includes either quinolone alone, or tetracycline and a third generation cephalosporin is associated with lower mortality. Penicillin alone is not particularly effective. We recommend treating patients who present with a clinical syndrome suggestive of $V$. vulnificus infection with a treatment regimen that includes a quinolone.

\section{Competing interests}

No authors have any commercial or other association that might pose a conflict of interest (e.g., pharmaceutical stock ownership, consultancy, advisory board membership, relevant patents, or research funding). The University of Western Sydney and the University of Sydney have no conflict of interest in the content and publication of this paper.

\section{Authors' contributions}

All authors participated in the conceptualisation of the research objectives and methods. KCW and SJW performed the coding of variables with free text fields using SNOMED-CT. GL managed and analysed the data. KCW, AB, and GL wrote the manuscript; and all authors reviewed and approved the manuscript.

\section{Acknowledgements}

We would like to thank Dr Hazel Dalton at the School of Rural Health University of Sydney for her assistance in the research grant application; Ms Anna Newton at the CDC for providing access to the COVIS dataset; and the University of Western Sydney (UWS) for providing an Early Career Research Grant to KCW; and Professor Annemarie Hennessy (Dean of the School of Medicine, University of Western Sydney) for her valuable comments to our manuscript.

\section{Declaration}

The content of this manuscript has not been presented in any meeting at the time of submission of this manuscript to the journal.

\section{Funding}

This work was supported by the University of Western Sydney (Conjoint Early Career Research Grant awarded to author KCW).

\section{Author details}

'Bathurst Rural Clinical School, School of Medicine, University of Western Sydney, PO Box 9008, Bathurst, NSW 2795, Australia. ${ }^{2}$ School of Rural Health, Sydney Medical School, University of Sydney, PO Box 1191, Orange 2800 NSW, Australia.

Received: 12 November 2014 Accepted: 21 May 2015

Published online: 11 June 2015 


\section{References}

1. Newton A et al. Increasing rates of vibriosis in the United States, 1996-2010: review of surveillance data from 2 systems. Clin Infect Dis. 2012:54 Suppl 5:S391-5.

2. Daniels NA. Vibrio vulnificus oysters: pearls and perils. Clin Infect Dis 2011:52(6):788-92

3. Centers for Disease Control and Prevention. National Cholera and Vibriosis Surveillance. 201427 June 2014]; Available from: http://www.cdc.gov/ nationalsurveillance/cholera-vibrio-surveillance.html.

4. Centers for Disease Control and Prevention. Vibrio Illness (Vibriosis). 201327 June 2014]; Available from: http://www.cdc.gov/vibrio/index.html.

5. Greenough 3rd W et al. Tetracycline in the treatment of cholera. Lancet. 1964:1(7329):355.

6. Nelson EJ et al. Antibiotics for both moderate and severe cholera. N Engl J Med. 2011;364(1):5-7.

7. Tang $\mathrm{HJ}$ et al. In vitro and in vivo activities of newer fluoroquinolones against Vibrio vulnificus. Antimicrob Agents Chemother. 2002;46(11):3580-4.

8. Horseman, M.A. and S. Surani. A comprehensive review of Vibrio vulnificus: an important cause of severe sepsis and skin and soft-tissue infection. Int J Infect Dis. 15(3): p. e157-66.

9. Purcell B et al. Effectiveness of antibiotics in preventing meningococcal disease after a case: systematic review. BMJ. 2004;328(7452):1339.

10. Dechet $\mathrm{AM}$ et al. Nonfoodborne Vibrio infections: an important cause of morbidity and mortality in the United States, 1997-2006. Clin Infect Dis. 2008;46(7):970-6.

11. International Health Terminology Standards Development Organisation. SNOMED CT. 201427 June 2014]; Available from: http://www.ihtsdo.org/ snomed-ct/.

12. Highly invasive new bacterium isolated from US east coast waters. JAMA, National Institutes of Health. 1984. 251(3): p. 323-5.

13. Morris Jr JG, Tenney J. Antibiotic therapy for Vibrio vulnificus infection. JAMA. 1985;253(8):1121-2.

14. Shaw, K.S., et al. Antimicrobial susceptibility of vibrio vulnificus and vibrio parahaemolyticus recovered from recreational and commercial areas of chesapeake bay and maryland coastal bays PLOS One, 2014. 9(2):e89616.

15. Linder JA et al. Fluoroquinolone prescribing in the United States: 1995 to 2002. Am J Med. 2005;118(3):259-68.

16. Wazana A. Physicians and the pharmaceutical industry: is a gift ever just a gift? JAMA. 2000;283(3):373-80

17. Chew LD et al. A physician survey of the effect of drug sample availability on physicians' behavior. J Gen Intern Med. 2000;15(7):478-83.

18. Bullen JJ et al. Hemochromatosis, iron and septicemia caused by Vibrio vulnificus. Arch Intern Med. 1991;151(8):1606-9.

19. Hlady WG, Klontz KC. The epidemiology of Vibrio infections in Florida, 1981-1993. J Infect Dis. 1996;173(5):1176-83.

20. Brennt CE et al. Growth of Vibrio vulnificus in serum from alcoholics: association with high transferrin iron saturation. J Infect Dis. 1991;164(5):1030-2.

21. Blake PA et al. Disease caused by a marine Vibrio Clinical characteristics and epidemiology. N Engl J Med. 1979;300(1):1-5.

22. Weis, K.E., et al. Vibrio illness in Florida, 1998-2007. Epidemiol Infect. 2011;139(4): p. 591-8.

\section{Submit your next manuscript to BioMed Central and take full advantage of:}

- Convenient online submission

- Thorough peer review

- No space constraints or color figure charges

- Immediate publication on acceptance

- Inclusion in PubMed, CAS, Scopus and Google Scholar

- Research which is freely available for redistribution 